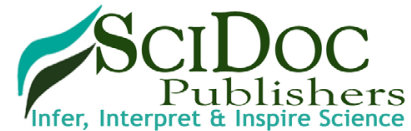

International Journal of Behavioral Research \& Psychology (IJBRP) ISSN 2332-3000

\title{
A Negative Feedback Between Democracy, Human Behavior and Achievement Of A Goal
}

Editorial

\author{
Aleksandar Zunjic \\ Professor, University of Belgrade, Belgrade, Serbia.
}

This paper aims to point out the negative effect that democracy can have on the achievement of social goals, based on the stimulation of a certain type of human behavior. It is common knowledge that democracy is a civilizational creation that forces decision-making based on a majority vote. In addition, democracy is characterized by freedom of expression, where each individual has the right to express their own opinion. Although these two characteristics of democracy sound promising and logical at first, they do not always give good results in practice. Moreover, in some cases, a democratic approach to achieving social goals can yield extremely poor outcomes. On the basis of a virtual example, which represents a simulation of a real situation, it has been shown how this is theoretically and practically possible.

\section{Example Of How Democracy Can Have A Nega- tive Influence On Human Behavior That Does Not Contribute To Achieving The Goal}

On the example of a virtual case study that represents a simulation of a real situation, it will be shown how democracy stimulates certain types of human behavior, which do not contribute to the achievement of a certain collective goal. In this regard, the realization of a completely new mode of transport, with the feature that the transport time is reduced by $90 \%$ with the simultaneous realization of large economic savings, is set as a goal. Suppose also that the final choice included 3 persons, who proposed one solution for the realization of this goal.

In the vast majority of cases, when it comes to technology and natural phenomena, only one solution in all sense meets all the criteria, that is, it affects the achievement of the goal in a direct, truly justified way. Let person A advocate such a solution. However, in some cases, the same solution can be reached in another, detour way. Although a solution can be reached in such a way, the whole procedure of achieving such a solution can take much longer, sometimes even years, or decades longer. Let person B advocate such a solution. Of course, there are also methods whose application will never lead to the achievement of the set goal. Let person $\mathrm{C}$ advocate such a proposal of a solution.

Given that these are solutions that will greatly affect the lives of all people and that the implementation of such solutions requires a large sum of money, let's assume that a referendum was called as a democratic form of expression of people, based on which the final solution will be chosen, and whose realization will be done in the future. Suppose all three persons exposed their version of the solution to a wide audience of people. Realistically, only a small number of people working in the area in question could understand (in whole or in part) the presentation of persons A, B and C. Suppose also that person $\mathrm{C}$ is already known to a large number of people, that he is an extroverted person who is a good speaker, has a high level of self-confidence and who knows how to attract an audience. Let person B is known to a much smaller number of people, whereby it possesses a lower level of self-confidence and relatively limited speaking skills and abilities, and who, in addition to all that, has relatively little experience in performing in front of a wide audience. In the end, let's assume that person A is completely unknown to the public, introverted, with a physical appearance that is "below average", relatively poorly dressed, with low self-confidence and speaking skills, without any experience in attracting audiences.

The question is, which proposal will the people vote for? Let us remind ourselves once again that voting is a form of people's behavior that is publicly stimulated and that it represents the supreme reflection of democracy. Given that the vast majority of people, at least $95 \%$, did not fully or partially understand the technical and scientific arguments of persons A, B and C, it is almost certain that they will vote for the person and the proposal that left the strongest impression on them. This means that person $\mathrm{C}$ will get the most votes for his proposal (for example 65\%), person B will get less (eg 30\%), while person A will get the least votes for his proposal (eg 5\%). So, based on the results of the democratic vote, the solution offered by person $\mathrm{C}$ will be chosen for the winning solution. For the realization of that solution, it will be necessary to invest a lot of work and money in the coming years.

*Corresponding Author

Aleksandar Zunjic,

Professor, University of Belgrade, Belgrade, Serbia.

E-mail: azunjic@mas.bg.ac.rs

Received: March 01, 2021

Published: March 02, 2021

Citation: Aleksandar Zunjic. A Negative Feedback Between Democracy, Human Behavior and Achievement Of A Goal. Int J Behav Res Psychol. 2021;09(01e):01-02. doi: http://dx.doi.org/10.19070/2332-3000-2100012e

Copyright: Aleksandar Zunjic ${ }^{\circ}$ 2021. This is an open-access article distributed under the terms of the Creative Commons Attribution License, which permits unrestricted use, distribution and reproduction in any medium, provided the original author and source are credited. 
However, let us remind that solution $\mathrm{C}$ is the wrong solution, which will never lead to the achievement of the goal. Besides, the damage is most likely double. Not only will the set goal with the chosen solution not be achieved, but the solution of person A will almost certainly be permanently rejected, because the people have already declared themselves about it.

\section{Conclusion}

Democracy greatly influences people's behavior. It can be seen as a stimulus for various forms of behavior, which can have a number of harmful consequences. For example, it stimulates the expression of views on various issues by people who sometimes haven't any knowledge about the problem, or have insufficient knowledge of the phenomenon they are talking about. Today's media, television, newspapers and other media are overwhelmed by the views of irrelevant persons on issues for which they are not competent, or have incomplete knowledge when it comes to a particular phenomenon. In that way, such persons, especially if they are known to the public on some other basis, largely shape public opinion with their attitudes, creating a false impression of the truth about a certain phenomenon in insufficiently informed, uncritical persons, and especially in the younger, inexperienced population.

Having in mind the above, it can be concluded that democracy is "a good servant, but a bad master". Democracy is a good servant in cases when it is used to inform the public about certain problems people are facing. However, democracy becomes a bad master in cases when it is used to make so-called "democratic solutions". Democratic solutions are those solutions that represent an inadequate compromise that takes into account the various views offered, among which there are also those that generally do not contribute to the achievement of the goal, or even make it difficult to achieve the goal. Such democratic solutions are sometimes good for creating a climate without conflicts or with fewer conflicts between different groups of people, but as such, they usually do not lead to a direct solution to a particular problem. Even worse is the situation when a democratic decision is made by a majority vote, where the majority advocates a solution that either does not lead to the fulfillment of the general goal, or leads to greater material or time investments that are necessary to achieve the goal.

The virtual case study described above reflects in a drastic but realistic way how democracy can influence making wrong decisions. However, less noticeable analog examples are found on a daily basis at the micro-level, in companies, institutions that approve scientific projects, etc. Modern society has accepted democracy as a magic wand for achieving social goals. Unfortunately, it has become a means of misuse in many cases. In modern society, it is not rare that in order to achieve a "democratic majority" are using bribery and blackmail. These are all forms of deviant behavior that arise as a result of the inadequate implementation of democracy. In order to prevent such forms of deviant behavior of people and to act preventively on making decisions that are harmful to society, it is necessary, in the time to come, to direct democracy in positive currents through education and new legal frameworks. 